\title{
A randomized Phase II clinical study of combining panitumumab and bevacizumab, plus irinotecan, 5-fluorouracil, and leucovorin (FOLFIRI) compared with FOLFIRI alone as second-line treatment for patients with metastatic colorectal cancer and KRAS mutation
}

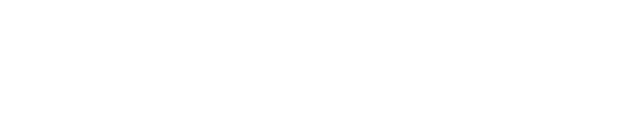

Yuguo Liu

Lijuan Luan

Xingli Wang

Department of Gastrointestinal Surgery, Shandong Tumor Hospital, Jinan, Shandong Province, People's Republic of China
Correspondence: Xingli Wang Department of Gastrointestinal Surgery, Shandong Tumor Hospital, 440 Jiyan Road, Jinan, Shandong Province 250II7, People's Republic of China Tel +8653I 67626285

Email wanggj122@aol.com
Background: This study investigated the efficacy and safety of a new treatment strategy of combining panitumumab and bevacizumab, plus irinotecan, 5-fluorouracil, and leucovorin (FOLFIRI) versus FOLFIRI alone as second-line chemotherapy for metastatic colorectal cancer (mCRC) patients with known V-Ki-ras2 Kirsten rat sarcoma viral oncogene (KRAS) mutation status.

Methods: Patients with mCRC who had known KRAS tumor status and unsuccessful previous oxaliplatin-based chemotherapy were included in the study. They were randomly assigned to two groups to receive panitumumab and bevacizumab plus FOLFIRI, or FOLFIRI alone. In panitumumab and bevacizumab plus FOLFIRI group, patients were given $4 \mathrm{mg} / \mathrm{kg}$ panitumumab and bevacizumab plus FOLFIRI every 2 weeks.

Results: In all, 65 patients were assigned to panitumumab and bevacizumab plus FOLFIRI group, and 77 to FOLFIRI alone group. For WT KRAS patients, the median progression-free survival (PFS) was 5.7 months (95\% confidence interval [CI], 2.4-7.5 months) for panitumumab and bevacizumab plus FOLFIRI and 3.8 months (95\% CI, 3.0-6.7 months) for FOLFIRI alone; median overall survival (OS) was 15.2 months (95\% CI, 8.9-19.7 months) for panitumumab and bevacizumab plus FOLFIRI and 11.0 months (95\% CI, 8.2-15.4 months) for FOLFIRI alone. For MU KRAS patients, median PFS was 5.1 months (95\% CI, 2.7-10.2 months) for panitumumab and bevacizumab plus FOLFIRI and 4.1 months (95\% CI, 2.5-8.4 months) for FOLFIRI alone; median OS was 12.8 months (95\% CI, 7.8-15.8 months) for panitumumab and bevacizumab plus FOLFIRI and 10.5 months (95\% CI, 6.1-15.3 months) for FOLFIRI alone. Grade 3 and 4 adverse events were associated with panitumumab and bevacizumab plus FOLFIRI but tolerable among patients.

Conclusion: Patients with mCRC can be safely and efficiently treated with second-line chemotherapy of combining panitumumab and bevacizumab plus FOLFIRI, despite their KRAS mutation status.

Keywords: metastatic colorectal cancer, panitumumab, bevacizumab, FOLFIRI, second-line chemotherapy

\section{Introduction}

Colorectal cancer (CRC) is one of the three most common cancers in men, and one of the two most common cancers in women worldwide. ${ }^{1}$ Every year, more than 1 million 
patients are diagnosed with CRC and most of them develop into metastatic colorectal cancer (mCRC). ${ }^{1,2}$ The standard single-agent chemotherapy for patients with mCRC typically uses regimen of 5-fluorouracil (5-FU) or leucovorin, oxaliplatin and folinic acid, 5-FU and irinotecan (FOLFIRI). ${ }^{3-5}$ Recently, as we gained advanced knowledge on the underlying mechanisms of mCRC, the combination chemotherapy including targeted regimens, such as aflibercept, regorafenib, cetuximab, panitumumab, and bevacizumab, into traditional chemo-reagents for $\mathrm{mCRC}$ had shown a significant progress on extending patients' long-term survival. ${ }^{6-10}$

The human homolog of V-Ki-ras2 Kirsten rat sarcoma viral oncogene (KRAS) is a GTPase protein that also acts as an oncogenic regulator. The mutation of KRAS is commonly found in various types of cancers, including mCRC. ${ }^{11-14}$ Studies demonstrated that KRAS mutation is highly associated with poor prognosis in patients with CRC. ${ }^{13-15}$ Panitumumab is an anti-epidermal growth factor receptor (EGFR) human monoclonal antibody, and has been applied in both first- and second-chemotherapy settings for patients with mCRC. ${ }^{16-20}$ However, clinical evidence showed mCRC patients with KRAS mutation, approximately 30\%-50\% of total patients with $\mathrm{mCRC}$, responded poorly to panitumumab chemotherapy. ${ }^{17,18}$ Moreover, a recent randomized Phase III trial of second-line chemotherapy demonstrated that panitumumab plus FOLFIRI was only able to improve progression-free survival (PFS), but not overall survival (OS) in $\mathrm{mCRC}$ patients. ${ }^{7}$

Bevacizumab is another human monoclonal antibody, thus has been utilized in target-specific chemotherapy for patients with mCRC. Unlike panitumumab, the target of bevacizumab is vascular endothelial growth factor (VEGF). It was suggested that KRAS protein might regulate the VEGF pathway to exert an angiogenic effect, ${ }^{21,22}$ and bevacizumab was shown to improve patients' OS and response rates (RRs) in irinotecan-based chemotherapy of mCRC. ${ }^{6,23}$ However, it is not clear whether mCRC patients with mutated KRAS would directly benefit from bevacizumab chemotherapy. ${ }^{24}$

Recently, two clinical studies demonstrated that combining panitumumab and bevacizumab plus FOLFIRI improved prognosis in patients with $\mathrm{mCRC}$ in second-line chemotherapy settings. ${ }^{25,26}$ However, these studies did not specify the relevance of KRAS mutation in combination chemotherapy. In the present study, we used a similar strategy of combining panitumumab and bevacizumab plus FOLFIRI as a second-line treatment option for patients with $\mathrm{mCRC}$, and examined the patients' response to treatment based on their KRAS mutation status.

\section{Patients and methods}

\section{Patients}

Eligible patients were between $\geq 18$ and $\leq 85$ years of age, having Eastern Cooperative Oncology Group (ECOG) performance status $\leq 2$, diagnosed with metastatic adenocarcinoma of colon or rectum with at least one unidimensionally measurable lesion $\geq 20 \mathrm{~mm}$ based on magnetic resonance imaging (MRI) or computerized tomography (CT) imaging. Patients had to have only one prior oxaliplatin- or 5-FU-based chemotherapy but still diagnosed with disease progression within 6 months of first-line treatment. Patients had to have adequate hematologic, renal, and hepatic functions, and without any prior anti-VEGF or anti-EGFR treatment for mCRC.

Patients were excluded if they had received prior antiEGFR or anti-VEGF therapy, had major surgery, hormonal therapy, immunotherapy within 4 weeks, or radiotherapy within 2 weeks of our study.

The protocol and treatment plan was approved by the Ethics Committee and Clinical Study Review Committee at Shandong Tumor Hospital. All patients provided signed consent forms before any procedures were conducted.

\section{Study design and treatment schedule}

This was an open-label, randomized, Phase II clinic study comparing the efficacy and safety of combining panitumumab and bevacizumab plus FOLFIRI. Patients were randomly stratified into two groups. In panitumumab and bevacizumab plus FOLFIRI group, patients were given $4 \mathrm{mg} / \mathrm{kg}$ panitumumab and $4 \mathrm{mg} / \mathrm{kg}$ bevacizumab plus FOLFIRI, every 2 weeks. Panitumumab and bevacizumab were administrated during a 90-minute infusion period before chemotherapy. In FOLFIRI alone group, patients were given FOLFIRI only $\left(180 \mathrm{mg} / \mathrm{m}^{2}\right.$ irinotecan, $400 \mathrm{mg} / \mathrm{m}^{2}$ racemic leucovorin by intravenous [IV] infusion on day 1 and FU $400 \mathrm{mg} / \mathrm{m}^{2}$ intravenous bolus on day 1, followed by $2,400 \mathrm{mg} / \mathrm{m}^{2}$ continuous infusion over days 1 and 2), every 2 weeks.

Responses were initially assessed by investigators, then confirmed by an independent radiologist blinded to the study, based on Response Evaluation Criteria in Solid Tumors (RECIST) every 8 weeks until disease progression. All patients were followed up on safety at least 45 days after the last study, and on survival every 90 days. During and after treatments, toxicity profiles were assessed for each patient. The adverse events (AEs) were graded according to National Cancer Institute Common Terminology Criteria for Adverse Events version 3.0. 


\section{KRAS determination}

KRAS mutation was determined by an allele-specific PCR method according to the previous study. ${ }^{27}$ Briefly, paraffinembedded tumor tissues were deparaffinized, and DNA was extracted. A KRAS mutation kit (DXS, Farnham, UK) was used to detect seven mutated sites on codons 12 and 13, Gly12Asp, Gly12Ala, Gly12Val, Gly12Ser, Gly12Arg, Gly12Cys, and Gly13Asp. An experienced statistician at Core Clinical Laboratory then performed blinded KRAS analysis to validate the PCR results.

\section{Statistical analysis}

The primary endpoints were RR, OS, and PFS. Secondary endpoints were AEs. Statistical analysis was performed with a windows-based SPSS software (version 11.0). Sample size calculations were conducted with the assumption of one-sided
$10 \% \alpha, 80 \%$ power, and a 1:1 randomization. OS and PFS were estimated using the Kaplan-Meier model with a confidence interval (CI) of 95\% for event-free probabilities and differences in probabilities, calculated by the Brookmeyer and Crowley method. Hazard ratios (HRs) were estimated by the Cox proportional hazards regression model.

\section{Results \\ Patients}

Between June 2010 and May 2014, there were a total of 142 patients who were eligible for the study. The CONSORT diagram is shown in Figure 1. Among all eligible patients, $65(46 \%)$ were assigned to panitumumab and bevacizumab plus FOLFIRI and 77 (54\%) to FOLFIRI alone. Of those patients, $126(89 \%)$ had test results of KRAS screening: 65 patients (52\%) having wild-type (WT) KRAS

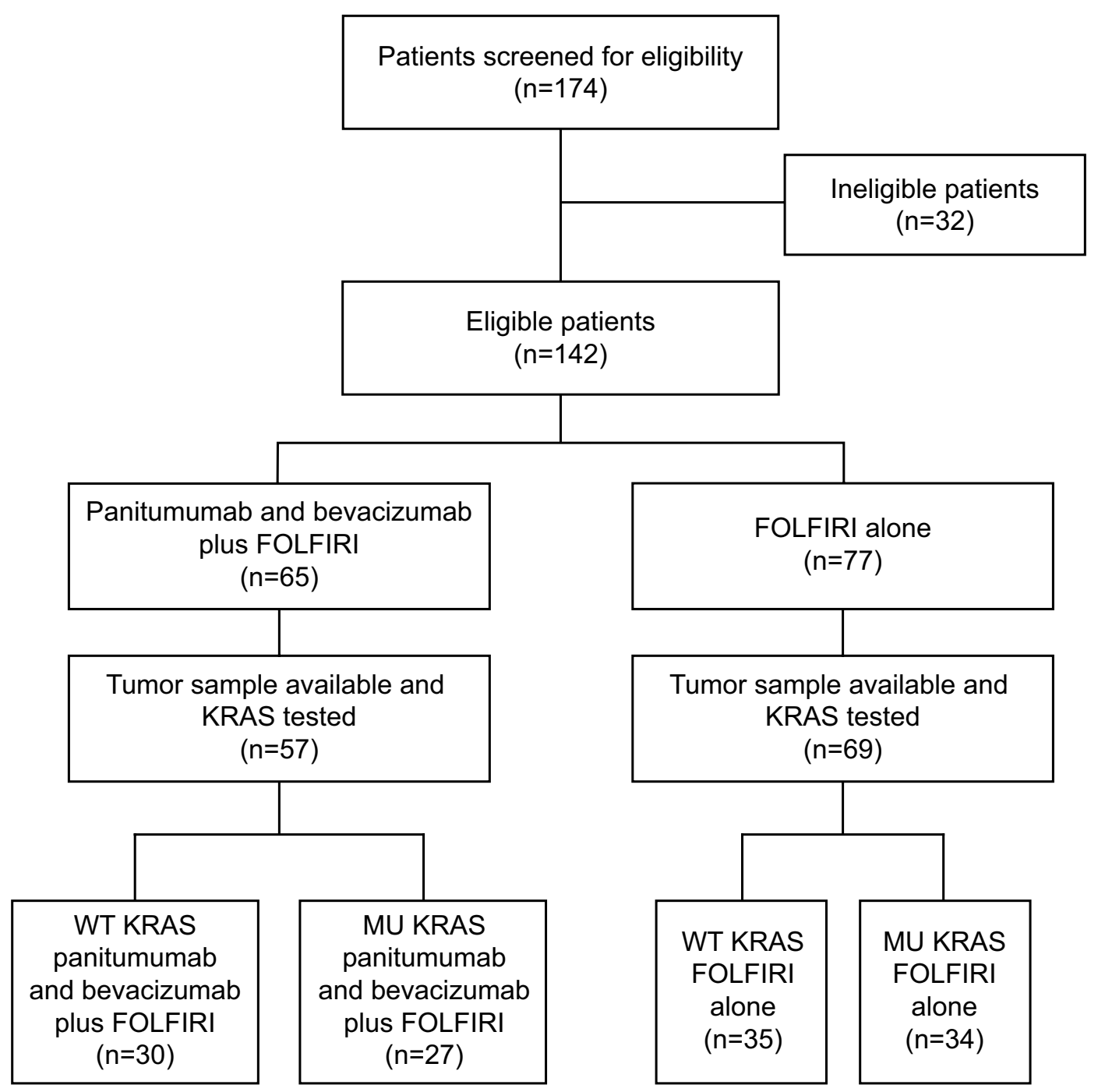

Figure I CONSORT diagram for flow of patients through the study.

Abbreviations: FOLFIRI, fluorouracil, leucovorin, and irinotecan; KRAS, V-Ki-ras2 Kirsten rat sarcoma viral oncogene; WT, wild-type; MU, mutant. 
tumors and 61 patients (48\%) having mutant (MU) KRAS tumors.

Baseline demographics and disease characteristics of the patients were stratified between those with WT and MU KRAS tumors, and balanced between two groups (panitumumab and bevacizumab plus FOLFIRI versus FOLFIRI alone), and balanced among (Table 1). For patients with WT KRAS tumors, the median ages were 59 years (21-82 years) in panitumumab and bevacizumab plus FOLFIRI group, and 62 years (25-80 years) in FOLFIRI alone group. For patients with MU KRAS tumors, the median ages were 61 years (22-79 years) in panitumumab and bevacizumab plus FOLFIRI group, and 60 years (29-85 years) in FOLFIRI alone group. For Eastern Cooperative Oncology Group performance status (ECOG PS), the majority of the patients had ECOG PS $<2$. For patients with WT KRAS tumors, 93\% of those in panitumumab and bevacizumab plus FOLFIRI group, and $91 \%$ of those in FOLFIRI alone group, had ECOG PS of 0 and 1, respectively. For patients with MU KRAS tumors, $93 \%$ of those in panitumumab and bevacizumab plus FOLFIRI group, and 94\% of those in FOLFIRI alone group, had ECOG PS of 0 and 1, respectively. In each group, for patients with either WT or MU KRAS tumors, about $60 \%$ of them had a primary tumor site in colon, and about $60 \%$ of them received first-line chemotherapy of FOLFOX.

\section{Efficacy}

PFSs and OSs were examined for patients with WT KRAS and MU KRAS tumors (Figure 2).

For patients with WT KRAS tumors, the median PFS was 5.7 months (95\% CI, 2.4-7.5 months) for panitumumab and bevacizumab plus FOLFIRI and 3.8 months $(95 \% \mathrm{CI}$, 3.0-6.7 months) for FOLFIRI alone. There was a significant improvement in PFS with panitumumab and bevacizumab plus FOLFIRI versus FOLFIRI alone (HR $=0.45 ; 95 \%$ CI, 0.22-0.64; $P<0.01$, Figure 2A). Median OS was 15.2 months (95\% CI, 8.9-19.7 months) for panitumumab and bevacizumab plus FOLFIRI and 11.0 months (95\% CI, 8.2-15.4 months) for FOLFIRI alone. There was also a significant improvement in OS with panitumumab and bevacizumab plus FOLFIRI versus FOLFIRI alone $(\mathrm{HR}=0.79$; 95\% CI, 0.47-0.89; $P<0.01$, Figure 2B).

For patients with MU KRAS tumors, the median PFS was 5.1 months (95\% CI, 2.7-10.2 months) for panitumumab and bevacizumab plus FOLFIRI and 4.1 months (95\% CI, 2.5-8.4 months) for FOLFIRI alone. There was a significant improvement in PFS with panitumumab and bevacizumab

Table I The demographics and disease characteristics of patients

\begin{tabular}{|c|c|c|c|c|c|c|c|c|}
\hline \multirow[t]{3}{*}{ Characteristics } & \multicolumn{4}{|c|}{ WT KRAS } & \multicolumn{4}{|c|}{ MU KRAS } \\
\hline & \multicolumn{2}{|c|}{$\begin{array}{l}\text { Panitumumab and } \\
\text { bevacizumab plus } \\
\text { FOLFIRI }(n=30)\end{array}$} & \multicolumn{2}{|c|}{$\begin{array}{l}\text { FOLFIRI alone } \\
(n=35)\end{array}$} & \multicolumn{2}{|c|}{$\begin{array}{l}\text { Panitumumab and } \\
\text { bevacizumab plus } \\
\text { FOLFIRI }(\mathbf{n}=\mathbf{2 7})\end{array}$} & \multicolumn{2}{|c|}{$\begin{array}{l}\text { FOLFIRI alone } \\
(n=34)\end{array}$} \\
\hline & No & $\%$ & No & $\%$ & No & $\%$ & No & $\%$ \\
\hline Sex, male & 18 & 60 & 22 & 63 & 17 & 63 & 20 & 59 \\
\hline \multicolumn{9}{|l|}{ Age (years) } \\
\hline Median & 59 & & 62 & & 61 & & 60 & \\
\hline Minimum & 21 & & 25 & & 22 & & 29 & \\
\hline Maximum & 82 & & 80 & & 79 & & 85 & \\
\hline \multicolumn{9}{|l|}{ ECOG PS } \\
\hline $0-1$ & 28 & 93 & 32 & 91 & 25 & 93 & 32 & 94 \\
\hline 2 & 2 & 7 & 3 & 9 & 2 & 7 & 2 & 6 \\
\hline \multicolumn{9}{|l|}{ Primary tumor } \\
\hline Colon & 19 & 63 & 21 & 60 & 18 & 67 & 20 & 59 \\
\hline Rectum & 11 & 27 & 14 & 40 & 9 & 23 & 14 & 41 \\
\hline \multicolumn{9}{|l|}{ Metastatic diseases } \\
\hline Liver only & 9 & 30 & 9 & 26 & 7 & 26 & 10 & 29 \\
\hline Other only & 3 & 10 & 4 & 11 & 3 & 11 & 2 & 6 \\
\hline Liver + other & 18 & 60 & 22 & 63 & 17 & 63 & 22 & 65 \\
\hline \multicolumn{9}{|c|}{ First-line chemotherapy } \\
\hline FOLFOX & 19 & 63 & 20 & 60 & 18 & 67 & 20 & 59 \\
\hline XELOX & 11 & 27 & 15 & 40 & 9 & 23 & 14 & $4 I$ \\
\hline
\end{tabular}

Abbreviations: WT, wild-type; KRAS, V-Ki-ras2 Kirsten rat sarcoma viral oncogene; FOLFIRI, fluorouracil, leucovorin, and irinotecan; MU, mutant; No, number; ECOG PS, Eastern Cooperative Oncology Group Performance Status. 

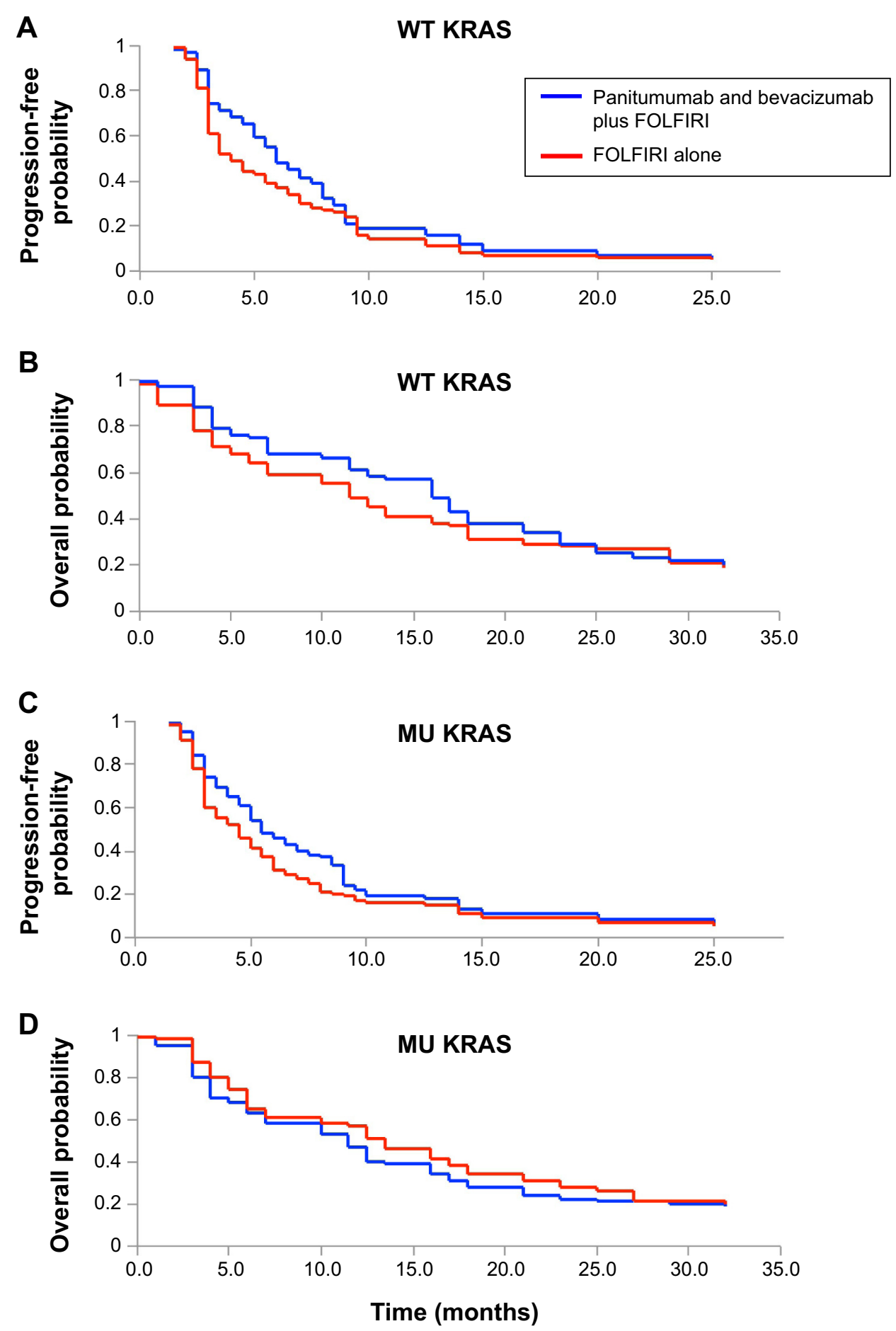

Figure 2 Progression-free survival (A) or overall survival (B) for wild-type (WT) KRAS, and progression-free survival (C) or overall survival (D) for mutant (MU) KRAS were examined.

Abbreviations: KRAS, V-Ki-ras2 Kirsten rat sarcoma viral oncogene; FOLFIRI, fluorouracil, leucovorin, and irinotecan.

plus FOLFIRI versus FOLFIRI alone $(\mathrm{HR}=0.65 ; 95 \% \mathrm{CI}$, $0.37-0.88 ; P<0.05$, Figure $2 \mathrm{C})$. Median OS was 12.8 months (95\% CI, 7.8-15.8 months) for panitumumab and bevacizumab plus FOLFIRI and 10.5 months (95\% CI, 6.1-15.3 months) for FOLFIRI alone. There was also a significant improvement in OS with panitumumab and bevacizumab plus FOLFIRI versus FOLFIRI alone (HR $=0.44$; 95\% CI, $0.19-0.61 ; P<0.05$, Figure 2D).

The RRs are shown in Table 2. For patients who had WT KRAS tumors, the objective response rate (OR) was $47 \%$ 
Table 2 Response rates of the patients

\begin{tabular}{|c|c|c|c|c|c|c|c|c|}
\hline \multirow[t]{3}{*}{ Assessment } & \multicolumn{4}{|c|}{ WT KRAS } & \multicolumn{4}{|c|}{ MU KRAS } \\
\hline & \multicolumn{2}{|c|}{$\begin{array}{l}\text { Panitumumab and } \\
\text { bevacizumab plus } \\
\text { FOLFIRI }(n=30)\end{array}$} & \multicolumn{2}{|c|}{$\begin{array}{l}\text { FOLFIRI alone } \\
(n=35)\end{array}$} & \multicolumn{2}{|c|}{$\begin{array}{l}\text { Panitumumab and } \\
\text { bevacizumab plus } \\
\text { FOLFIRI ( } \mathrm{n}=\mathbf{2 7})\end{array}$} & \multicolumn{2}{|c|}{$\begin{array}{l}\text { FOLFIRI alone } \\
(n=34)\end{array}$} \\
\hline & No & $\%$ & No & $\%$ & No & $\%$ & No & $\%$ \\
\hline Objective response & 14 & 47 & 9 & 26 & 12 & 44 & 10 & 29 \\
\hline Complete response & I & 3 & 0 & 0 & 0 & 0 & 0 & 0 \\
\hline Partial response & 13 & 44 & 9 & 26 & 12 & 44 & 10 & 29 \\
\hline Stable disease & 5 & 17 & 3 & 9 & 8 & 30 & 8 & 24 \\
\hline
\end{tabular}

Abbreviations: WT, wild-type; KRAS, V-Ki-ras2 Kirsten rat sarcoma viral oncogene; FOLFIRI, fluorouracil, leucovorin, and irinotecan; MU, mutant; No, number.

(95\% CI, 32\%-59\%) in panitumumab and bevacizumab plus FOLFIRI group, significantly better than $26 \%$ (95\% CI, $13 \%-41 \%)$ in FOLFIRI alone group $(P<0.001)$. For patients who had MU KRAS, the OR was 44\% (95\% CI, 22\%-53\%) in panitumumab and bevacizumab plus FOLFIRI group, also significantly better than $29 \%(95 \% \mathrm{CI}, 19 \%-55 \%)$ in the FOLFIRI alone group $(P<0.05)$.

\section{Safety}

Grade 3 and 4 AEs for the patients with both WT and MU KRAS tumors are listed in Table 3. The incidence rates of grade 3 and 4 AEs in the WT KRAS patients in the panitumumab and bevacizumab plus FOLFIRI group and FOLFIRI alone group were $93 \%$ and $60 \%$, respectively. The incidence rates of grade 3 and 4 AEs in the MU KRAS patients in the panitumumab and bevacizumab plus FOLFIRI group and FOLFIRI alone group were $93 \%$ and $56 \%$, respectively.

Moreover, despite KRAS tumor status, more patients experienced antibody-associated grade 3 and 4 AEs, including hypertension, bleeding, and proteinuria. For patients with WT KRAS tumors, the incidence rates of severe hypertension, bleeding, and proteinuria were $17 \%, 23 \%$, and $13 \%$, respectively, in panitumumab and bevacizumab plus FOLFIRI group. Those were higher than the rates of $6 \%, 3 \%$, and $0 \%$, in FOLFIRI alone group $(P<0.01)$. For patients with MU KRAS tumors, the incidence rates of severe hypertension, bleeding, and proteinuria were $15 \%, 22 \%$, and $19 \%$, respectively, in panitumumab and bevacizumab plus FOLFIRI group, also significantly higher than the rates of $3 \%, 6 \%$, and $3 \%$, in FOLFIRI alone group $(P<0.01)$.

\section{Discussion}

There have been two recent clinical studies, both in China, analyzing the treatment effects of combining anti-EGFR mAb and anti-VEGF mAb for patients with previously treated mCRC. ${ }^{25,26}$ However, neither of them correlated their results with KTAS mutation. Our study is the first to prospectively examine the efficacy and safety of combining panitumumab and bevacizumab according to the KRAS tumor status in patients with prior but failed first-line chemotherapy. And we

Table 3 Grade 3 and 4 adverse events (AEs) of the patients

\begin{tabular}{|c|c|c|c|c|c|c|c|c|}
\hline \multirow[t]{3}{*}{ AEs } & \multicolumn{4}{|c|}{ WT KRAS } & \multicolumn{4}{|c|}{ MU KRAS } \\
\hline & \multicolumn{2}{|c|}{$\begin{array}{l}\text { Panitumumab and } \\
\text { bevacizumab plus } \\
\text { FOLFIRI }(n=30)\end{array}$} & \multicolumn{2}{|c|}{$\begin{array}{l}\text { FOLFIRI alone } \\
(n=35)\end{array}$} & \multicolumn{2}{|c|}{$\begin{array}{l}\text { Panitumumab and } \\
\text { bevacizumab plus } \\
\text { FOLFIRI ( } n=27) \\
\end{array}$} & \multicolumn{2}{|c|}{$\begin{array}{l}\text { FOLFIRI alone } \\
(n=34)\end{array}$} \\
\hline & No & $\%$ & No & $\%$ & No & $\%$ & No & $\%$ \\
\hline Patients with AEs & 28 & 93 & 21 & 60 & 25 & 93 & 19 & 56 \\
\hline Skin toxicity & 12 & 40 & 5 & 18 & 20 & 74 & 5 & 15 \\
\hline Neutropenia & 7 & 23 & 3 & 9 & 8 & 30 & 2 & 6 \\
\hline Diarrhea & 5 & 17 & 2 & 6 & 5 & 19 & 1 & 3 \\
\hline Hypokalemia & 4 & 13 & I & 3 & 5 & 19 & 0 & 0 \\
\hline Mucositis & 5 & 17 & I & 3 & 4 & 15 & 2 & 6 \\
\hline Pulmonary embolism & 4 & 13 & I & 3 & 4 & 15 & 0 & 0 \\
\hline Dehydration & 2 & 7 & 0 & 0 & 2 & 7 & 0 & 0 \\
\hline Hypertension & 5 & 17 & 2 & 6 & 4 & 15 & 1 & 3 \\
\hline Bleeding & 7 & 23 & I & 3 & 6 & 22 & 2 & 6 \\
\hline Proteinuria & 4 & 13 & 0 & 0 & 5 & 19 & 1 & 3 \\
\hline
\end{tabular}

Abbreviations: WT, wild-type; KRAS, V-Ki-ras2 Kirsten rat sarcoma viral oncogene; FOLFIRI, fluorouracil, leucovorin, and irinotecan; MU, mutant; No, number. 
demonstrated that, despite tumor status, panitumumab and bevacizumab plus FOLFIRI was effective in improving PFS and OS in patients with both WT and MU KRAS tumors.

In our study, for FOLFIRI alone treatment, the median PFSs were 3.8 months for patients with WT KRAS tumors and 4.1 months for patients with MU KRAS tumors; the median OSs were 11.0 months for patients with WT KRAS tumors and 10.5 months for patients with MU KRAS tumors; and ORs were $26 \%$ for patients with WT KRAS tumors and $29 \%$ for patients with MU KRAS tumors. These data were consistent with previous studies while using a similar second-line chemotherapy setting of biweekly treatment of FOLFIRI, reporting PFS ranging from 2 to 7 months, OS ranging from 8 to 15 months and disease-control rates ranging from $20 \%$ to $50 \% .^{25,28,29}$ Thus, it suggests that, even the sample size of our study was smaller than previous ones, it was unlikely to confound the results of our study due to sampling errors.

Most importantly, we demonstrated that, despite the KRAS status, both WT and MU patients may benefit from the second-line chemotherapy combining panitumumab and bevacizumab. For patients with WT KRAS tumors, absolute improvements were observed in both PFS (5.7 months for panitumumab and bevacizumab plus FOLFIRI versus 3.8 months for FOLFIRI alone) and OS (15.2 months for panitumumab and bevacizumab plus FOLFIRI versus 11.0 months for FOLFIRI alone. Similar results were observed in MU KRAS patients, with absolute improvements in PFS (5.1 months versus 4.1 months) and OS (12.8 months versus 10.5 months), comparing panitumumab and bevacizumab plus FOLFIRI group and FOLFIRI alone group. This result is very encouraging as previous clinic trials showed that only those patients with WT KRAS tumors responded positively to panitumumab chemotherapy. ${ }^{17,18,27}$ However, it was not clear, at least from current study, whether the combined regimen of bevacizumab was solely responsible for the survival improvements in patients with MU KRAS tumors, or adding bevacizumab into panitumumab resulted in synergetic effects on patients with MU KRAS tumors. Thus, future clinical studies comparing treatment effects between combination chemotherapy (panitumumab and bevacizumab) and mono-agent chemotherapy (panitumumab or bevacizumab) would help to optimize the treatment strategy of second-line chemotherapy for patients with mCRC.

Overall, our study clearly demonstrated that combination chemotherapy of panitumumab and bevacizumab plus FOLFIRI was effective in treating patients with mCRC in second-line setting with tolerable toxicity profiles. Patients with both WT and MU KRAS tumors can benefit from this combination chemotherapy.

\section{Disclosure}

The authors report no conflicts of interest in this work.

\section{References}

1. Jemal A, Siegel R, Xu J, Ward E. Cancer statistics, 2010. CA Cancer J Clin. 2010;60:277-300.

2. Ciombor KK, Berlin J. Targeting metastatic colorectal cancer - present and emerging treatment options. Pharmgenomics Pers Med. 2014; 7: 137-144.

3. Cunningham D, Pyrhonen S, James RD, et al. Randomised trial of irinotecan plus supportive care versus supportive care alone after fluorouracil failure for patients with metastatic colorectal cancer. Lancet. 1998;352:1413-1418.

4. Rougier P, Van Cutsem E, Bajetta E, et al. Randomised trial of irinotecan versus fluorouracil by continuous infusion after fluorouracil failure in patients with metastatic colorectal cancer. Lancet. 1998;352: $1407-1412$.

5. Fuchs CS, Marshall J, Mitchell E, et al. Randomized, controlled trial of irinotecan plus infusional, bolus, or oral fluoropyrimidines in firstline treatment of metastatic colorectal cancer: results from the BICC-C Study. J Clin Oncol. 2007;25:4779-4786.

6. Hurwitz H, Fehrenbacher L, Novotny W, et al. Bevacizumab plus irinotecan, fluorouracil, and leucovorin for metastatic colorectal cancer. New Engl J Med. 2004;350:2335-2342.

7. Peeters M, Price TJ, Cervantes A, et al. Randomized phase III study of panitumumab with fluorouracil, leucovorin, and irinotecan (FOLFIRI) compared with FOLFIRI alone as second-line treatment in patients with metastatic colorectal cancer. J Clin Oncol. 2010;28:4706-4713.

8. Van Cutsem E, Kohne CH, Lang I, et al. Cetuximab plus irinotecan, fluorouracil, and leucovorin as first-line treatment for metastatic colorectal cancer: updated analysis of overall survival according to tumor KRAS and BRAF mutation status. J Clin Oncol. 2011;29:2011-2019.

9. Macarulla T, Sauri T, Tabernero J. Evaluation of aflibercept in the treatment of metastatic colorectal cancer. Expert Opin Biol Ther. 2014; 14:1493-1505.

10. Sastre J, Argiles G, Benavides M, et al. Clinical management of regorafenib in the treatment of patients with advanced colorectal cancer. Clin Transl Oncol. 2014.

11. Timar J. The clinical relevance of KRAS gene mutation in non-smallcell lung cancer. Curr Opin Oncol. 2014;26:138-144.

12. Bos JL. Ras oncogenes in human cancer: a review. Cancer Res. 1989; 49:4682-4689.

13. Andreyev HJ, Norman AR, Cunningham D, et al. Kirsten ras mutations in patients with colorectal cancer: the 'RASCAL II' study. Br JCancer. 2001; 85:692-696.

14. Esteller M, Gonzalez S, Risques RA, et al. K-ras and p16 aberrations confer poor prognosis in human colorectal cancer. J Clin Oncol. 2001; 19:299-304.

15. Imamura $Y$, Lochhead $P$, Yamauchi M, et al. Analyses of clinicopathological, molecular, and prognostic associations of KRAS codon 61 and codon 146 mutations in colorectal cancer: cohort study and literature review. Mol Cancer. 2014;13:135.

16. Cohn AL, Shumaker GC, Khandelwal P, et al. An open-label, single-arm, phase 2 trial of panitumumab plus FOLFIRI as second-line therapy in patients with metastatic colorectal cancer. Clin Colorectal Cancer. 2011; 10:171-177.

17. Mitchell EP, Piperdi B, Lacouture ME, et al. The efficacy and safety of panitumumab administered concomitantly with FOLFIRI or Irinotecan in second-line therapy for metastatic colorectal cancer: the secondary analysis from STEPP (Skin Toxicity Evaluation Protocol With Panitumumab) by KRAS status. Clin Colorectal Cancer. 2011;10:333-339. 
18. Hocking CM, Price TJ. Panitumumab in the management of patients with KRAS wild-type metastatic colorectal cancer. Therap Adv Gastroenterol. 2014;7:20-37.

19. Kohne CH, Hofheinz R, Mineur L, et al. First-line panitumumab plus irinotecan/5-fluorouracil/leucovorin treatment in patients with metastatic colorectal cancer. J Cancer Res Clin Oncol. 2012;138:65-72.

20. Berlin J, Posey J, Tchekmedyian S, et al. Panitumumab with irinotecan/ leucovorin/5-fluorouracil for first-line treatment of metastatic colorectal cancer. Clin Colorectal Cancer. 2007;6:427-432.

21. Rak J, Yu JL, Kerbel RS, Coomber BL. What do oncogenic mutations have to do with angiogenesis/vascular dependence of tumors? Cancer Res. 2002;62:1931-1934.

22. Mizukami Y, Kohgo Y, Chung DC. Hypoxia inducible factor-1 independent pathways in tumor angiogenesis. Clin Cancer Res. 2007; 13:5670-5674.

23. Heinemann V, Hoff PM. Bevacizumab plus irinotecan-based regimens in the treatment of metastatic colorectal cancer. Oncology. 2010;79: $118-128$.

24. Hurwitz HI, Yi J, Ince W, Novotny WF, Rosen O. The clinical benefit of bevacizumab in metastatic colorectal cancer is independent of K-ras mutation status: analysis of a phase III study of bevacizumab with chemotherapy in previously untreated metastatic colorectal cancer. Oncologist. 2009;14:22-28.
25. Xie S, Han G, Fan Z, He L, Xu W, Qin Z. Safety and efficacy of secondline treatment with folinic acid, 5-fluorouracil and irinotecan (FOLFIRI) in combination of panitumumab and bevacizumab for patients with metastatic colorectal cancer. Med Oncol. 2014;31:35.

26. Liang HL, Hu AP, Li SL, Liu JY. Combining bevacizumab and panitumumab with irinotecan, 5-fluorouracil, and leucovorin (FOLFIRI) as second-line treatment in patients with metastatic colorectal cancer. Med Oncol. 2014;31:976.

27. Amado RG, Wolf M, Peeters M, et al. Wild-type KRAS is required for panitumumab efficacy in patients with metastatic colorectal cancer. J Clin Oncol. 2008;26:1626-1634.

28. Fuchs CS, Moore MR, Harker G, Villa L, Rinaldi D, Hecht JR. Phase III comparison of two irinotecan dosing regimens in second-line therapy of metastatic colorectal cancer. J Clin Oncol. 2003;21:807-814.

29. Muro K, Boku N, Shimada Y, et al. Irinotecan plus S-1 (IRIS) versus fluorouracil and folinic acid plus irinotecan (FOLFIRI) as second-line chemotherapy for metastatic colorectal cancer: a randomised phase 2/3 non-inferiority study (FIRIS study). Lancet Oncol. 2010;11:853-860.
OncoTargets and Therapy

\section{Publish your work in this journal}

OncoTargets and Therapy is an international, peer-reviewed, open access journal focusing on the pathological basis of all cancers, potential targets for therapy and treatment protocols employed to improve the management of cancer patients. The journal also focuses on the impact of management programs and new therapeutic agents and protocols on

\section{Dovepress}

patient perspectives such as quality of life, adherence and satisfaction. The manuscript management system is completely online and includes a very quick and fair peer-review system, which is all easy to use. Visit http://www.dovepress.com/testimonials.php to read real quotes from published authors. 